\title{
Anthrax case ignites new forensics field
}

The messy tragedy surrounding the 2001 US anthrax attacks and the suicide of chief suspect Bruce Ivins has thrown the emergent field of microbial forensics into the spotlight. The forensic techniques proved vital in allowing the Federal Bureau of Investigation (FBI) to make its case that the anthrax used in the attacks came from a particular sample in Ivins's lab.

Ivins died before facing charges, but had his case reached trial it would have been a major test for the discipline, which barely existed before 2001.

Microbial forensics traces the origin of a biological agent using a range of biochemical analyses, including genomic sequencing and protein and carbohydrate fingerprinting. The discipline has its roots in military and intelligence communities, and was used successfully in court as far back as 1998, in the trial of a doctor in Louisiana who intentionally infected his former mistress with HIV. But the field took off during the anthrax investigation with an

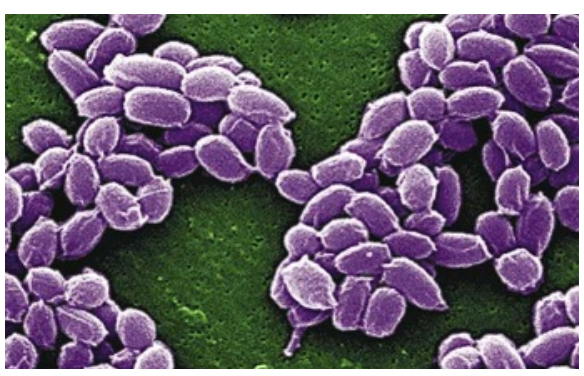

Anthrax spores were forensically analysed.

influx of government funding and the advent of cheaper, faster sequencing technology.

Microbial forensics is likely to have an increasing role beyond biocrime. "The big thing that's going to be useful out of all this is its application to molecular epidemiology," says Paul Keim, a molecular geneticist at Northern Arizona University in Flagstaff, who worked with the FBI investigation. For example, microbial forensics could be used to pinpoint the source of food- or water-borne pathogens. Referring to the recent US Salmonella outbreak, Keim says, "If the same thing happens in a year, I would expect to see hundreds of Salmonella genomes sequenced."

The field could also be important in lawsuits over hospital-acquired infections, says microbiologist Abigail Salyers of the University of Illinois at Urbana-Champaign. People have sued hospitals for millions, claiming they contracted methicillin-resistant Staphylococcus aureus (MRSA) as patients. Microbial forensics could be used to trace an infection to its source and ascertain whether it was acquired at a particular hospital.

Microbiologists will need to be confident of their technique - forensic analysis requires unusual rigour. In a journal publication, it is usually understood that further work is needed, says Keim. "In court, you don't have that luxury," he says. "You have to get it right the first time."

Amber Dance 